\title{
Novel Mental Retardation-Epilepsy Syndrome Linked to Xp21.1-p11.4
}

\author{
Peter Hedera, MD, ${ }^{1}$ David Alvarado, BS, ${ }^{1}$ Ahmad Beydoun, MD, ${ }^{1}$ and John K. Fink, MD $^{1,2}$
}

\begin{abstract}
We evaluated a kindred with X-linked mental retardation and epilepsy. Seven affected males with mild to moderate mental retardation developed seizures (primarily generalized, tonic-clonic, and atonic) that began on average at 6.8 months of age (range, 4 to 14 months). These patients did not have a history of infantile spasms. There were no dysmorphic features. Other than mental retardation, the neurological examination was unremarkable, with exception of 2 affected subjects who had mild generalized rigidity and ataxia. We identified tight linkage to a group of markers on Xp21.1-p11.4. A maximum two-point LOD score of +3.83 at $\theta=0$ was obtained for markers DXS8090, DXS1069, DXS8102, and DXS8085. This locus spans 7.7cM between DXS1049 and DXS8054 and does not overlap the locus for $\mathrm{X}$-linked West syndrome. The tetraspanin gene, implicated in nonspecific mental retardation, is mapped to this region. We sequenced the tetraspanin coding sequence in subjects with X-linked mental retardation and epilepsy and did not identify disease-specific mutations. The syndrome we describe, designated X-linked mental retardation and epilepsy, is clinically and genetically distinct from X-linked West syndrome and other X-linked mental retardation-epilepsy syndromes.
\end{abstract}

Ann Neurol 2002;51:45-50

Mental retardation (MR) is a common disorder that affects males with disproportionate frequency. It is estimated that X-linked inheritance is responsible for $25 \%$ of all MR. ${ }^{1}$ X-linked mental retardation (XLMR) is clinically heterogeneous. XLMR is classified as nonspecific when reduced intelligence quotient (IQ) is the only clinical abnormality, and as syndromic when recognizable metabolic abnormalities, neurological deficits, or dysmorphic features are present. A total of 202 XLMR conditions have been genetically mapped, and 33 XLMR genes have been identified. ${ }^{2}$

Seizures are features of several XLMR disorders. The combination of seizures and mental retardation sometimes represents metabolic abnormalities or developmental abnormalities of brain formation. ${ }^{3-7}$ At other times, however, epilepsy and XLMR can occur without diagnostic laboratory or neuroimaging abnormalities. West syndrome characterized by MR, a type of myoclonic seizure known as infantile spasm (IS), and a unique encephalographic pattern (hypsarrhythmia), is usually sporadic or secondary to underlying brain disturbance, such as tuberous sclerosis. ${ }^{8}$ However, in several families, West syndrome has been transmitted as an X-linked disorder (OMIM \# 308350) and has been mapped to a 7-cM locus on Xp21.3-Xp22.1., ${ }^{9,10}$

We evaluated a kindred in which epilepsy and MR were found to be transmitted as an X-linked recessive trait. We designate this condition X-linked mental retardation-epilepsy syndrome (XMRE). Seizure pattern and degree of mental retardation distinguish this syndrome from West syndrome. We performed genetic linkage analysis and identified a tight linkage between XMRE and polymorphic microsatellite markers spanning a 7.7-cM locus on Xp21.1-p11.4 that does not overlap the West syndrome locus on Xp21.3-22.1. ${ }^{10}$

Tetraspanin (TM4SF2), a recently identified gene that causes MR, was mapped to Xp11.4. ${ }^{11} \mathrm{We}$ sequenced the TM4SF2 coding region and found no mutations in affected subjects from our XMRE kindred. This finding indicates that TM4SF2 coding sequence mutations do not cause Xp21.3-22.1-linked XMRE.

\section{Patients and Methods}

\section{Clinical, and Laboratory Studies}

We evaluated 20 members of a nonconsanguinous North American kindred of Polish descent (Fig 1). Informed consent was obtained from each subject as specified by the University of Michigan Institutional Review Board. Subjects were diagnosed as affected or unaffected with MR and epilepsy on the basis of history and neurological examination and of review of the medical records at the time of blood
From the ${ }^{1}$ Department of Neurology, University of Michigan; ${ }^{2} \mathrm{Ge}-$ riatric Research, Education, and Clinical Center, Veterans Affairs Medical Center, Ann Arbor, MI.

Received May 30, 2001, and in revised form Sep 4, 2001. Accepted for publication Sep 4, 2001.
Address correspondence to Dr Fink, 5214 Cancer Center Geriatrics Center Building, 1500 E. Medical Center Drive, Ann Arbor, MI 48109-0940. E-mail: jkfink@umich.edu 


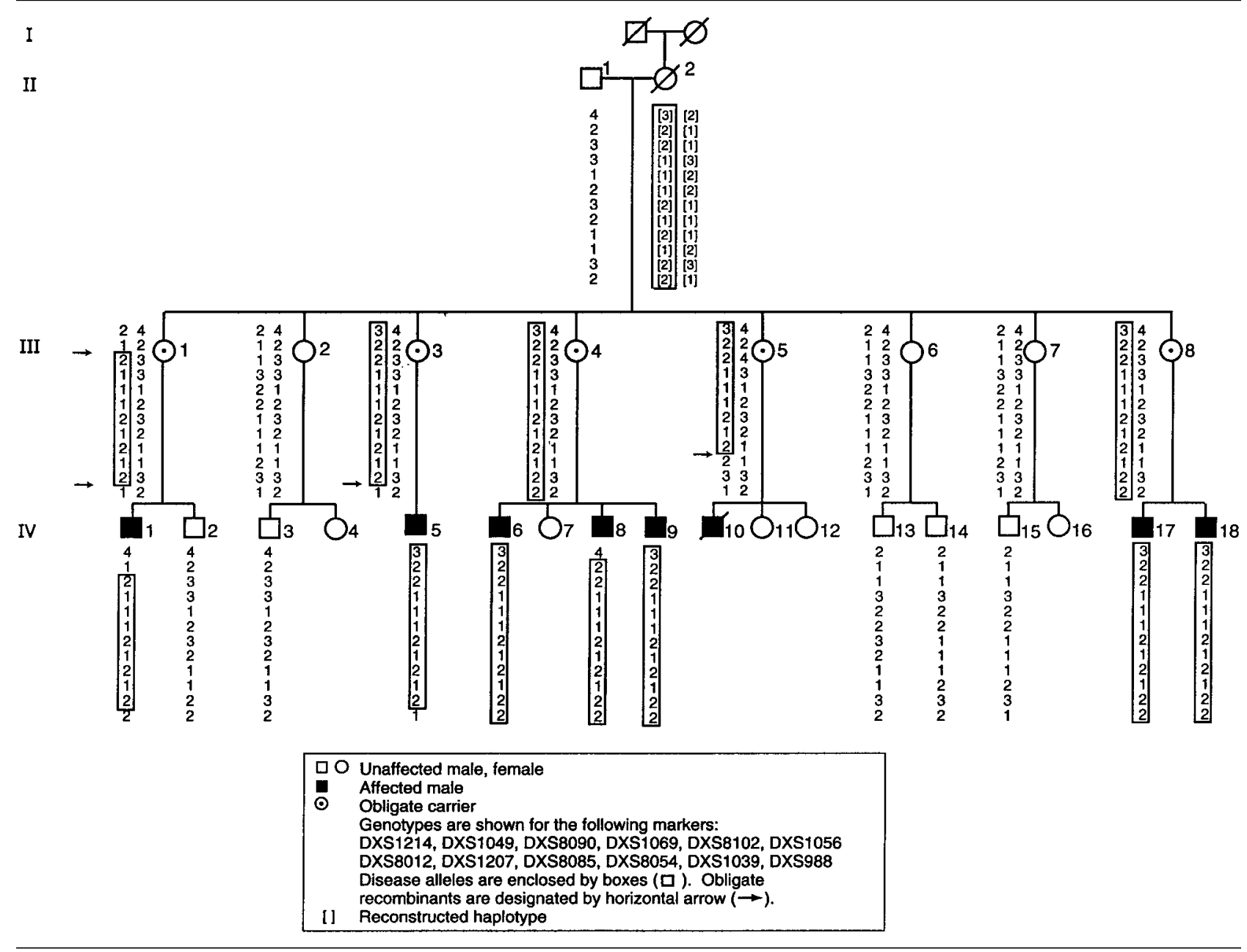

Fig 1. X-linked mental retardation-epilepsy (XMRE) pedigree.

collection and before genotyping. Information about age of seizure onset and seizure semiology was obtained by interviewing the parents of affected subjects and reviewing medical records. Electroencephalographic (EEG) tracings were examined for subjects IV-1 and IV-5; EEG reports were reviewed for subjects IV-6 and IV-8. Electromyography (EMG) and nerve conduction studies were performed on subjects IV-5 and IV-9; magnetic resonance imaging (MRI) of the brain were performed on subjects IV-1, IV-5, IV-8, IV-9, and IV-17; fragile $\mathrm{X}$ analysis was performed on subjects IV-1, IV-5, IV-9, IV-17, and IV-18; and urine metabolic screen, venous lactate and pyruvate, and plasma verylong-chain fatty acid analysis obtained on subjects IV-1, IV-5, IV-9, and IV-17. High-resolution prometaphase karyotype was obtained for subject IV-5. Formal psychometric assessment was obtained for subjects IV-1, IV-5, IV-9, and IV-18.

\section{Genetic Linkage Analysis}

Pedigree analysis (see Fig 1) was consistent with transmission of the disorder as a monogenic X-linked recessive trait. Therefore, we analyzed 37 microsatellite polymorphisms spaced 5 to $2020 \mathrm{cM}$ throughout the X-chromosome for linkage with the disorder. Microsatellite DNA polymorphisms were analyzed as previously described. ${ }^{12}$

We performed genetic linkage analysis using the unaffected grandfather (subject II-1, Fig 1), his 8 daughters (subjects III-1 through III- 8 ), 5 of whom were considered obligate maternal carriers (subjects III-1, III-3, III-4, III-5, and III-8); 7 living affected subjects (subjects IV-1, IV-5, IV-6, IV-8, IV-9, IV-17, and IV-18), and 5 living unaffected subjects (IV-2, IV-3, IV-13, IV-14, and IV-15). Two-point linkage analyses were performed with the MLINK subroutine of the LINKAGE program, using an X-linked model of disease inheritance and disease allele frequency of 0.001 . We assigned genetic penetrance of 0.90 for lod-score calculations. Lod scores were calculated with marker allele frequencies assumed to be equal because the small family size prevented accurate estimation of marker allele frequency within this family.

\section{Analysis of the Tetraspanin Gene}

Intronic primers were designed for seven TM4SF2 exons based on the published TM4SF2 DNA sequence (www3. ncbi.nlm.nih.gov) using Lasergene software. TM4SF2 exons were amplified from gDNA samples of all affected and unaf- 
Table 1. Clinical Analysis of XMRE Subjects

\begin{tabular}{|c|c|c|c|c|c|c|c|c|}
\hline & IV-1 & IV -5 & IV-6 & IV-8 & IV-9 & IV-10 & IV-17 & IV -18 \\
\hline Age (yr) & 11 & 23 & 23 & 19 & 15 & 2 (deceased) & 6 & 3 \\
\hline $\begin{array}{l}\text { Age of seizure } \\
\text { onset (mo) }\end{array}$ & 4 & 10 & 6 & 4 & 6 & 4 & 14 & 6 \\
\hline Type of seizures & GTC & GTC & GTC & GTC & GTC & GTC & GTC & GTC \\
\hline & Drop attacks & Drop attacks & & & & & Drop attacks & Drop attacks \\
\hline $\begin{array}{l}\text { Seizure free (in- } \\
\text { terval) }\end{array}$ & $\begin{array}{l}\text { Not seizure } \\
\text { free }\end{array}$ & $\begin{array}{l}\text { Not seizure } \\
\text { free }\end{array}$ & Yes $(10$ yr $)$ & Yes $(2 \mathrm{yr})$ & Yes (3 yr) & $\begin{array}{l}\text { Not seizure } \\
\text { free }\end{array}$ & $\begin{array}{l}\text { Not seizure } \\
\text { free }\end{array}$ & $\begin{array}{l}\text { Not seizure } \\
\text { free }\end{array}$ \\
\hline $\begin{array}{l}\text { Current seizure } \\
\text { frequency }\end{array}$ & $3-4 / \mathrm{mo}$ & $2-5 / \mathrm{mo}$ & N/A & N/A & N/A & N/A & $0-2 / \mathrm{mo}$ & $0-2 / \mathrm{mo}$ \\
\hline $\begin{array}{l}\text { Current anti- } \\
\text { convulsants }\end{array}$ & CLZ, PHT & $\begin{array}{l}\text { CLZ, LTG, } \\
\text { TPM }\end{array}$ & N/A & N/A & VPA & N/A & VPA & VPA \\
\hline $\begin{array}{c}\text { Anticonvulsants } \\
\text { used in past }\end{array}$ & $\begin{array}{l}\text { CBZ, LTG, } \\
\text { TPM, VPA, } \\
\text { PB, FBM }\end{array}$ & $\begin{array}{l}\text { PB, CBZ, } \\
\text { VPA, PHT }\end{array}$ & $\begin{array}{l}\text { VPA, LTG, } \\
\text { PB, PHT }\end{array}$ & $\begin{array}{l}\text { VPA, LTG, } \\
\text { PHT }\end{array}$ & $\begin{array}{l}\text { VPA, PB, CBZ, } \\
\text { PHT }\end{array}$ & $\begin{array}{l}\text { VPA, PB, } \\
\text { PHT }\end{array}$ & $\mathrm{PB}, \mathrm{PHT}$ & PB, PHT \\
\hline
\end{tabular}

$\mathrm{XMRE}=\mathrm{X}$-linked mental retardation-epilepsy; CLZ = clonazepam; PHT $=$ phenytoin; VPA $=$ valproate; LTG $=$ lamotrigine; TPM $=$ topiramate; $\mathrm{CBZ}=$ carbamazepine; $\mathrm{PB}=$ phenobarbital; FBM = felbamate; GTC = generalized tonic-clonic seizures.

fected subjects. Polymerase chain reaction (PCR) products were purified through sephadex G-50 columns and sequenced using the ABI PRISM dRhodamine Terminator Cycle Sequencing Ready Reaction and the ABI PRISM 3100 Genetic Analyzer (PE Applied Biosystems, Foster City, CA). Each exon was sequenced in both directions and DNA sequence analyzed using SeqMan software (DNA Star, Boston, MA).

\section{Results}

\section{Clinical Features of X-Linked Mental Retardation,} Epilepsy Syndrome

PEDIGREE ANALYSIS AND EXAMINATION OF UNAFFECTED SUBJECTS. The 72-year-old grandfather (subject II-1; see Fig 1) and his 8 daughters, including obligate carriers (subjects III-1, III-3, III-4, III-5, and III-8; see Fig 1), had a normal neurological examination and no history of seizures, developmental delay, or learning disabilities. The lowest education level achieved was high school completion. The patient's wife (subject II-2) reportedly had normal intelligence and no history of seizures. She died at age 55 years from an unspecified cardiac cause. A review of the extended family on her side did not show any history of seizures or MR.

Obligate maternal carriers (subjects III-1, III-3, III-4, III-5, and III-8; see Fig 1) had 7 affected sons, 5 unaffected daughters (IV-4, IV-7, IV-11, IV-12, and IV-16; see Fig 1), and 5 unaffected sons (subjects IV-2, IV-3, IV-13, IV-14, and IV-15; see Fig 1). These progeny ranged in age from 4 to 25 years. Unaffected sons and daughters had normal developmental milestones and normal neurological examinations.

DEVELOPMENTAL MILESTONES. Affected subjects had normal gestation, labor, and delivery. Delay in achieving motor milestones was reported for subjects IV-1, IV-6, IV-9, and IV-17, who were unable to walk independently until 18 to 24 months of age. All affected subjects had delayed speech acquisition (range 16 to 36 months of age). Each affected subject had a normal hearing test. There was no history of psychomotor regression after the onset of seizures.

Seizures were the first evidence of neurological abnormality in all affected subjects and began between four and 14 months of age (average age of seizure onset was 6.8 months) (Table 1). Descriptions of seizure pattern were consistent with generalized tonic-clonic seizures and drop attacks consisting of brief atonic or myoclonic seizures with loss of posture. Parents of affected boys did not observe focal neurological signs before generalized seizures. Seizure frequency was variable and ranged from 20 seizures per day to several seizures per month. There were no episodes of status epilepticus. Parents were asked explicitly whether seizure pattern conformed to that of infantile spasms. There was no description of such seizures in any patient.

Various anticonvulsants had been used as monotherapy or in combination (see Table 1). All but one affected subject experienced reduction in seizure frequency with age; subject IV-5 despite having only two to three seizures annually as a teenager, experienced significant increase in his seizure frequency at age 20 years despite compliance with medications. Improved seizure control was achieved after substituting topiramate and lamotrigine for carbamazepine.

At evaluation, 3 subjects (IV-6, age 23 years; IV-8, age 19 years; IV-9, age 15 years) were seizure free for 10,2 , and 3 years, respectively. Anticonvulsants had been discontinued for two of these subjects (IV-6 and IV-8; see Fig 1).

Subject IV-10 developed generalized seizures, similar to his cousins at the age of 4 months. He died at age 2 years during sleep and his sudden death was attributed to a seizure. Autopsy report was not available.

MENTAL RETARDATION. For each affected subject, cognitive impairment was first evident between ages 24 and 36 months. Affected subjects were classified as 
having trainable mental impairment and required a special education. Formal assessment with Wechsler abbreviated scale of intelligence for subject IV-5 showed verbal IQ of 60 , performance IQ of 56, and full-scale IQ of 54; subjects IV-8 scores were 50, 57, 53 and subjectss IV-18 52, 56, and 54, respectively. Psychological assessment of subject IV-1 at age 8 years 10 months, using the MacArthur Communicative Development Inventory showed performance at 9- to 16month-old levels. ${ }^{13}$

GENERAL PHYSICAL EXAMINATION. The general physical examination showed a normal head circumference, height, and weight (50 to $75 \%$ for age) for each subject, except subject IV-8, who was mildly obese. No dysmorphic features were noted in any affected subject.

NEUROLOGICAL EXAMINATION. All subjects were able to perform simple calculations, name common objects and follow two- or three-step commands. There was no aphasia or dysarthria. Subject IV-8 had occasional echolalia. The remainder of the neurological examination of subjects IV-1, $-5,-6,-17$, and -18 was notable only for hyporeflexia in upper and lower extremities. Although parents described affected children as clumsy, dysmetria and dysdiadochokinesis were absent. Subject IV-5, who received clonazepam, topiramate, and lamotrigine, had mildly abnormal tandem gait without any additional abnormalities.

SCOLIOSIS AND GAIT DISTURBANCE WERE PRESENT IN TWO SUBJECTS. Two affected subjects (IV-8 and IV-9) had scoliosis, progressive gait disturbance, and bilateral pes planovalgus. There was no apparent change in intellectual abilities, speech or dexterity of either of these subjects with progressive gait disturbance. A third affected brother in this sibship (IV-6) had seizures and MR but did not have scoliosis or gait disturbance. Scoliosis in subject IV-9 was managed conservatively, but his brother (IV-8) benefited from corrective surgery. At age 8 years, brothers IV-8 and IV-9 developed gait disturbance described as dragging their feet and frequent falls. Subject IV-8 was still able to walk independently at age 19 years. His younger brother (IV-9) began to require a wheelchair at age 10 years. At age 15 years, he was able to transfer but required support of two persons to walk. His gait displayed adduction of both knees and both ankles. Neurological examination of both brothers demonstrated mild rigidity on lower extremities without cogwheeling. Deep tendon reflexes were hypoactive, with the exception of absent Achilles deep tendon reflexes (both subjects had prominent bilateral pes planovalgus). Plantar response was flexor bilaterally. Heel-to-shin testing showed a slight dysmetria. Subject IV-9 was treated with oral baclofen $(\leqslant 40 \mathrm{mg} /$ day $)$ and L-dopa $(\$ 500 \mathrm{mg} /$ day $)$ without benefit.

\section{Laboratory and Radiological Features}

LABORATORY EVALUATIONS. Laboratory evaluations performed for subjects IV-1, $-5,-9$, and -17 indicated no abnormal findings (see Patients and Methods).

ELECTROENCEPHALOGRAPHY. Each affected subject had at least one EEG. Interictal EEG recordings for subject IV-1 demonstrated frequent bursts of generalized $2-\mathrm{Hz}$ spike and wave and polyspike discharges with frontocentral amplitude predominance. Photic stimulation did not provoke EEG changes. Two generalized tonic-clonic seizures and one myoclonic seizure were recorded during closed-circuit video-EEG study of subject IV-5. Generalized polyspike and wave discharges were present before each seizure. Diffuse interictal background slowing and disorganization with generalized polyspike and slow wave discharges and multifocal independent sharp wave discharges were also noted. EEG studies performed at other institutions on other affected subjects showed only nonspecific generalized slowing.

NEUROIMAGING, ELECTROMYOGRAPHY, AND NERVE CONDUCTION STUDIES. MRI of the brain (subjects IV-1, IV-5, IV-8, IV-9, and IV-17) was normal. EMG and nerve conduction studies (obtained for subjects IV-5 and IV-9) were normal.

\section{Genetic Linkage Analysis}

IDENTIFICATION OF XMRE LOCUS ON XP21.1-P11.4. We observed linkage of the disorder to a group of microsatellite polymorphisms mapped to Xp21.1-p11.4 (Table 2). Using the assumption that genetic penetrance was 0.90 , the maximum two-point lod score was $+3.83(\theta=0)$ for markers DXS8090 (AFM338xa5), DXS1069 (AFM240wa9), DXS8102 (AFMa062xa9), and DXS8085 (AFM289wf5). Four additional markers, DXS1207 (AFM269yc), DXS1069 (AFM240wa), DXS8102 (AFMa062xa9), and DXS1056 (AFM$198 \mathrm{vb12}$ ) also yielded lod scores of greater than +1.0 (see Table 2). Haplotype analysis (Fig 1) revealed obligate recombinants (subjects III-5 and III-1; see Fig 1) for centromeric and telomeric flanking markers DXS8054 (AFMb291yc9) and DXS1049 (AFM$155 \mathrm{ze} 1)$, respectively; separated by $7.7 \mathrm{cM}$.

EXCLUSION OF WEST SYNDROME LOCUS ON XP21.3-P22.1. A locus for West syndrome has been mapped to Xp21.3-p22.1 between DXS274 and AHC. ${ }^{10}$ We tested linkage of the disorder in our kindred to polymorphic microsatellite markers mapped to this locus and observed significantly negative two-point lod 
Table 2. Two-Point LOD Scores Identify XMRE Locus on Xp21.3-Xp22.1

\begin{tabular}{|c|c|c|c|c|c|c|c|c|}
\hline \multicolumn{2}{|l|}{ Marker } & \multirow{2}{*}{$\begin{array}{c}\text { Penetrance } \\
0.9\end{array}$} & \multirow{2}{*}{$\begin{array}{c}\theta=0.001 \\
-3.07\end{array}$} & \multirow{2}{*}{$\begin{array}{c}0.05 \\
-0.29\end{array}$} & \multirow{2}{*}{$\begin{array}{l}0.10 \\
0.32\end{array}$} & \multirow{2}{*}{$\frac{0.20}{0.62}$} & \multirow{2}{*}{$\begin{array}{l}0.30 \\
0.50\end{array}$} & \multirow{2}{*}{$\begin{array}{l}0.40 \\
0.22\end{array}$} \\
\hline DXS1214 & AFM283wg9 & & & & & & & \\
\hline DXS1049 & AFM155ze1 & 0.9 & -0.92 & 0.57 & 0.68 & 0.60 & 0.38 & 0.15 \\
\hline DXS8090 & AFM338xa5 & 0.9 & 3.83 & 3.50 & 3.16 & 2.41 & 1.58 & 0.68 \\
\hline DXS1069 & AFM240wa9 & 0.9 & 3.83 & 3.50 & 3.16 & 2.41 & 1.58 & 0.68 \\
\hline DXS8102 & AFMa062xa9 & 0.9 & 1.47 & 1.32 & 1.17 & 0.84 & 0.50 & 0.18 \\
\hline DXS1056 & AFM198vb12 & 0.9 & 0.92 & 2.24 & 2.22 & 1.84 & 1.26 & 0.57 \\
\hline DXS8012 & AFMa124xd9 & 0.9 & 3.83 & 3.50 & 3.16 & 2.41 & 1.58 & 0.68 \\
\hline DXS1207 & AFM269ус5 & 0.9 & 2.11 & 1.96 & 1.78 & 1.42 & 0.99 & 0.48 \\
\hline DXS8085 & AFM289wf5 & 0.9 & 3.83 & 3.50 & 3.16 & 2.41 & 1.58 & 0.68 \\
\hline DXS8054 & AFMb291yc9 & 0.9 & -1.17 & 0.07 & 0.24 & 0.27 & 0.18 & 0.06 \\
\hline
\end{tabular}

$\mathrm{XMRE}=\mathrm{X}$-linked mental retardation-epilepsy.

scores (DXS1202 [AFM260ye5]-5.55 and DXS7106 [AFM333wf1] -1.89 at $\theta=0$ ). These results indicate that the disorder in our kindred is not linked to the West syndrome locus on Xp21.3-p22.1 (Fig 2).

Fig 2. Schematic representation of the X-linked mental retardation-epilepsy (XMRE) and West syndrome loci.

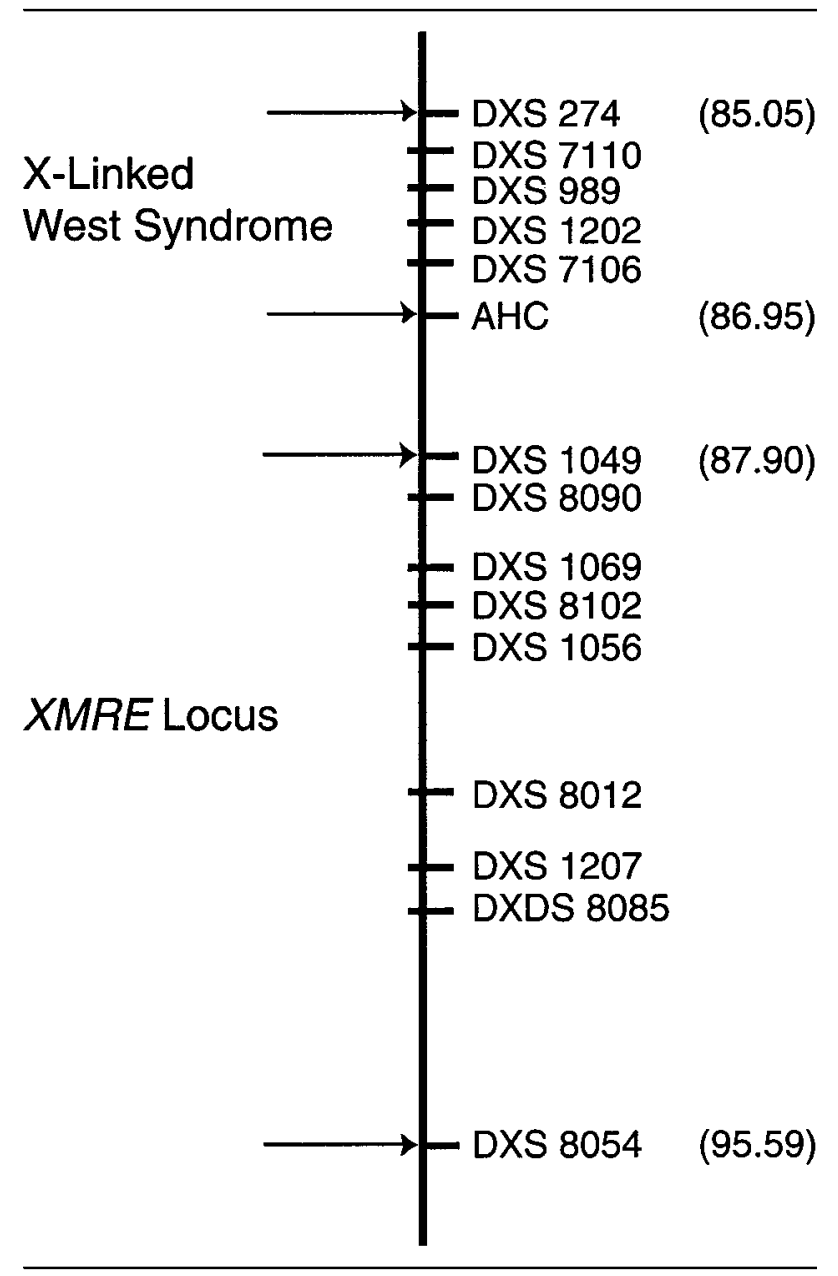

EXCLUSION OF TETRASPANIN CODING SEQUENCE MUTATION AS THE CAUSE OF XMRE. We sequenced each of the seven exons of TM4SF2 and did not identify coding sequence mutations in affected subjects.

\section{Discussion}

We describe an X-linked syndrome of mental retardation and epilepsy; and report identification of the locus for this XMRE syndrome on chromosome Xp21.1$\mathrm{p} 11.4$. We show that this locus is distinct from the X-linked West syndrome locus by testing genetic linkage to markers with this locus and observing several obligate recombinants.

EEG characteristics and degree of MR in our patients are distinct from males with X-linked West syndrome. Seizures in affected subjects in this family were consistent with symptomatic generalized epilepsy. None had infantile spasms or hypsarrhythmia, features that characterize West syndrome. ${ }^{8}$ West syndrome is also associated with arrested psychomotor development after seizure onset, typically resulting in severe mental retardation. In contrast, our patients did not exhibit significant regression after the development of epilepsy and had only mild to moderate mental retardation.

Thirty-one families with nonspecific mental retardation (MRX 4, 5, 7-15, 17, 18, 20-22, 26, 29, 31, 33, 38, 39, 43-45, and 50-52) have been linked to the chromosome $\mathrm{Xp}$ region that partially or completely overlaps the XMRE locus we identified. ${ }^{2}$ It is probable that a limited number of genes account for mental retardation in these unrelated families. Seizures were a constant feature in only one of these families, however (designated MRX38). ${ }^{14}$ Affected males developed seizures in this family in childhood, but the age of onset varied significantly (from 2 to 9 years) and epilepsy was relatively mild; none of these subjects had documented infantile spasms. Our subjects differ from MRX38 subjects because macrocephaly was a specific distinctive feature present in every symptomatic MRX38 sub- 
ject. ${ }^{14}$ We did not observe head circumference above the $98 \%$ in any affected subjects from the XMRE family presented in this study. Absence of macrocephaly, more severe epilepsy, and earlier age of seizure onset suggest that XMRE is distinct from other types of nonspecific X-linked mental retardation linked to Xp region.

Eight genes that cause nonspecific mental retardation have been identified, one of which, tetraspanin (TM4SF2), was mapped to the XMRE locus. ${ }^{11,15}$ The TM4SF2 gene was identified by positional cloning in a mentally retarded female patient who had an X;2 balanced translocation. TM4SF2 was interrupted by this translocation. Subsequently, TM4SF2 coding sequence point mutations were found in 2 small families with nonspecific mental retardation. Even though none of the patients with identified TM4SF2 mutations had seizures, this gene was an important candidate gene for XMRE. Nonetheless, sequencing the TM4SF2 coding region did not identify any coding sequence mutations in XMRE subjects. We cannot exclude, however, the possibility that disturbance in transcriptional regulation or mRNA splicing of this gene could underlie XMRE.

Identified genes causing XLMR are a heterogeneous group, but most of these genes are involved in signaling pathways. ${ }^{15}$ Several genes implicated in XLMR belong to the family of guanine nucleotide exchange factors for Rho GTPase, with putative roles in regulation of neuronal plasticity and cytoskeletal organization. ${ }^{16}$ Other XLMR genes, including TM4SF2, are transmembrane proteins that interact with extracellular molecules and participate in signaling pathways. ${ }^{11}$ Another recognized cause of seizures and MR are abnormalities in neuronal migration. ${ }^{17}$ Even though neuroimaging did not indicate any obvious migration defects, genes controlling neuronal migration remain important candidate genes as a cause of XMRE. Given the size $(7.7 \mathrm{cM})$ of the XMRE locus, identifying the XMRE gene may require ascertaining additional XMRE kindreds and reduction of the locus. Discovering the XMRE gene will advance our knowledge of the causes and, ultimately, treatment for MR and epilepsy.

This research is supported by grants from the Muscular Dystrophy Association, Veterans Affairs Merit Review, and the National
Institutes of Health (NINDS R01NS33645, R01NS36177, R01NS38713) to J.K.F. and the University of Michigan General Clinical Research Center (GCRC 1573).

\section{References}

1. Herbst DS, Miller JR. Non-specific X-linked mental retardation III: the frequency in British Columbia. Am J Med Genet 1980; 7:461-469.

2. Chiurazzi P, Hamel BC, Neri G. XLMR genes: update 2000. Eur J Hum Genet 2001;71-81.

3. Matsumoto N, Leventer RJ, Kuc JA, et al. Mutation analysis of the DCX gene an genotype/phenotype correlation in subcortical band heterotopia. Eur J Hum Genet 2001;9:5-12.

4. Pettigrew AL, Jackson LG, Ledbetter DH. New X-linked mental retardation disorder with Dandy-Walker malformation, basal ganglia disease, and seizures. Am J Med Genet 1991;38: 200-207.

5. Neidich JA, Nussbaum RL, Packer RJ, et al. Heterogeneity of clinical severity and molecular lesions in Aicardi syndrome. J Pediatr 1990;116:911-917.

6. Menkes JH. Kinky hair disease. Pediatrics 1972;50:181-182.

7. Brown GK, Otero LJ, LeGris M, Brown RM. Pyruvate dehydrogenase deficiency. J Med Genet 1994;31:875-879.

8. Chugani HT. Infantile spasms. Curr Opin Neurol 1995;8: $139-144$.

9. Claes S, Devriendt K, Lagae L, et al. The X-linked infantile spasms syndrome (MIM 308350) maps to Xp11.4-Xpter in two pedigrees. Ann Neurol 1997;42:360-364.

10. Bruyere H, Lewis MES, Wood S, et al. Confirmation of linkage in X-linked infantile spasms (West syndrome) and refinement of the disease locus to Xp21.3-Xp22.1. Clin Genet 1999;55: 173-181.

11. Zemni R, Bienvenu T, Vinet MC, et al. A new gene involved in X-linked mental retardation identified by analysis of X;2 balanced translocation. Nature Genet 2000;24:167-170.

12. Hedera P, Rainier S, Alvarado D, et al. Novel locus for autosomal dominant hereditary spastic paraplegia on chromosome 8q. Am J Hum Genet 1999;64:563-569.

13. Arriaga RI, Fenson L, Cronan T, Pethick SJ. Scores on the MacArthur communicative development inventory of children from low- and middle-income families. Appl Psycholinguist 1998;19:209-223.

14. Schultz CK, Ives EJ, Chalifoux M, et al. Regional localization of an X-linked mental retardation gene to Xp21.1-Xp22.13. Am J Med Genet 1996;64:89-96.

15. Gecz J, Mulley J. Genes for cognitive function: developments on the X. Genome Res 2000;10:157-163.

16. Hall A. Rho GTPases and the actin cytoskeleton. Science 1998; 279:509-514.

17. Allen KM, Walsh CA. Genes that regulate neuronal migration in the cerebral cortex. Epilepsy Res 1999;36:143-154. 